\title{
Liderazgo directivo y condiciones para la innovación en escuelas chilenas: el que nada hace, nada teme*
}

\author{
Leadership and management conditions for innovation in Chilean schools: \\ nothing to hide, nothing to fear
}

\author{
Condições de liderança e gestão de inovação em escolas chilenas: \\ nada agora, nada a temer
}

\author{
Francisco Leal-Soto, ${ }^{a}$ Mario Albornoz Hernández, ${ }^{b}$ María Isabel Rojas Parada ${ }^{c}$ \\ Departamento de Ciencias Sociales sede Esmeralda, Universidad de Tarapacá \\ Telf.: (56)572425775. Correo electrónico: fleal@uta.cl \\ Universidad de Tarapacá \\ Telf.: (56)582205792. Correo electrónico: malbornozh@gmail.com \\ Universidad de Tarapacá \\ Telf.: (56)582205792. Correo electrónico: mirojasp@gmail.com
}

\begin{abstract}
RESUMEN
Hoy la innovación es un imperativo en los sistemas educativos y la gestión educativa es crucial en el modo en que se desarrollan los procesos en las organizaciones. La relación entre liderazgo e innovación ha sido poco estudiada, en particular en el contexto chileno. Dentro de los elementos de gestión se ha identificado el liderazgo, describiéndose tres tipos con efectos diferentes: transaccional, transformacional y pasivo-evitativo. Se administró cuestionarios de liderazgo directivo y condiciones para la innovación a 127 profesores y profesoras de 14 establecimientos secundarios de Iquique, Chile. Predominaron los liderazgos transformacional y transaccional, que también mostraron alta correlación entre sí y correlacionaron positivamente con condiciones para la innovación. El liderazgo pasivo-evitador correlacionó negativamente con condiciones para la innovación. Tolerancia hacia el fracaso y disponibilidad de recursos fueron las condiciones menos frecuentes. Se discuten estos resultados a la luz de elementos de la cultura escolar y la cultura nacional chilena.
\end{abstract}

Palabras clave: innovación, liderazgo, gestión directiva, enseñanza secundaria, cultura escolar.

ABSTRACT
Today innovation is an imperative in education systems, and management is crucial in how the processes develop in organizations. The relationship between leadership and innovation has been little studied, particularly in the Chilean context. Among elements of management, leadership has been identified as relevant, and three types with different effects have been described: transactional, transformational and passive-avoidant. Questionnaires of managerial leadership and conditions for innovation were administered to 127 teachers from 14 secondary schools in Iquique, Chile. High transformational and transactional leadership were observed, which also show high correlation with each other and with conditions for innovation. The passive-avoidant leadership negatively correlated with conditions for innovation. Tolerance to failure and resources availability were the least frequent conditions. These results are discussed in light of elements of school culture and the Chilean national culture.

Key words: innovation, leadership, executive management, secondary education, school culture.

Este artículo es una reelaboración del trabajo desarrollado por los autores Mario Albornoz y María Isabel Rojas, bajo la dirección del primer autor, para la obtención del grado de Magíster en Educación en el Departamento de Educación de la Universidad de Tarapacá. 


\section{INTRODUCCIÓN}

El rol directivo fue un gran ausente en las políticas educacionales chilenas hasta fines del siglo XX. A comienzos de los años noventa, el estatuto docente privilegió la recuperación del alicaído rol de los maestros, aunque iniciando un tímido viraje que marcó un progresivo reconocimiento a las funciones del director y el equipo directivo, hasta llegar al énfasis en el liderazgo directivo puesto en la ley general de educación, pasando por el marco de la buena dirección, hito que marcó un paso crítico hacia el liderazgo pedagógico (Núñez, Weinstein y Muñoz, 2010). Ello, reconociendo un hecho que la investigación pone cada vez más de relieve: que entre las variables de la escuela el liderazgo directivo es el elemento más importante solo después de la influencia del profesor, a la hora de explicar los resultados académicos (Horn y Marfán, 2010). La investigación también muestra que la percepción de la influencia del rol directivo por parte de los actores escolares es polarizada: se la percibe como gestiones con buena o con mala influencia, traduciéndose esta percepción en exaltación o desvalorización (Tapia et al., 2011). Asimismo, los teóricos de la gestión educativa contemporánea ponen el liderazgo en el centro de los procesos de mejoramiento institucional (Riveros, 2012).

Por su parte, la innovación es reconocida como base de la economía actual, y el liderazgo ligado a la innovación es considerado uno de los factores decisivos para la supervivencia de las organizaciones (Turbay, 2013). En tal sentido, Chile corre aún con desventaja, aunque sigue siendo el país más competitivo en América Latina, se compara desfavorablemente con los países de la OCDE, y sus indicadores asociados a innovación son sistemáticamente mucho más bajos que sus indicadores de competitividad globales (Schwab, 2013). Entre las causas de ello, Cruz (2008) menciona la escasez de capital humano para la innovación, problema que comienza, a su juicio, en la baja calidad de la educación escolar. Esto nos lleva a plantear el tema de la innovación en la escuela, que tiene, al menos, dos formas de abordarse: la primera y más evidente para el sentido común, cómo incorporar la innovación al curriculum, es decir, cómo enseñar a ser innovador a fin de que las nuevas generaciones puedan hacer frente al cambiante, desafiante y competitivo mundo que enfrentarán; y la segunda, menos evidente, pero sin la cual resulta infructuoso plantearse la primera, cómo hacer de la innovación una práctica pedagógica; es decir, cómo incorporar o desarrollar dinámicas escolares que permitan innovar en las prácticas instruccionales. Es en esta segunda forma en la que se sitúa el trabajo que presentamos.

En este sentido, García (2008) puntualiza que la innovación es un desafío institucional para las organizaciones y las personas en ellas, que involucra sus valores y compromete su capacidad organizativa, constituyendo, ante todo, un reto para los directivos. En el específico ámbito de las organizaciones educativas, Restrepo y Restrepo (2012) destacan la complejidad de la tarea directiva actual, marcada por una multiplicidad de frentes y por un contexto de cambio de época caracterizado por la globalización, la complejidad y el dinamismo, enfatizando en la necesidad del liderazgo de innovación. Si los directivos son claves en las instituciones educativas, hoy están, además, llamados a ser líderes que generen condiciones y promuevan la innovación al interior de sus escuelas.

Aunque innovación es un término cada vez más utilizado en el ámbito educativo, es un término complejo y polisémico, por lo que no siempre que es utilizado se está hablando de lo mismo (Barraza, 2007). Asimismo, suele utilizarse en compañía de otros términos, como innovación educacional, innovación educativa o, incluso, innovación con efecto educativo 
(Blanco y Messina, 2000). Barraza (2005) se refiere a la innovación educativa como un proceso complejo que concierne a la selección, organización y uso creativo de los elementos de gestión institucional, curricular y didáctica, generalmente en respuesta a un problema o necesidad que exige respuestas integrales; enfatiza que la innovación ha de ser vivida como experiencia personal y como resultado de un proceso colaborativo. Vega (1994 Cit. en Blanco y Messina, 2000), por su parte, subraya el carácter de cambio sistémico de la innovación educativa, y la relevancia de la participación crítica de todos los actores involucrados para su éxito. Por ello, las características de los directivos y del clima que fomentan en la comunidad educativa cobran relevancia como condiciones facilitadoras de la innovación educativa.

Se han identificado múltiples condiciones organizacionales como necesarias para o facilitadoras de la innovación. Modelos de gestión como el de la European Foundation For Quality Management en Europa (Ferrando y Granero, 2005) o el Baldrige Performance Excellence Program ${ }^{1}$ en los Estados Unidos de Norteamérica destacan la importancia de valorar el conocimiento más allá de los ámbitos específicos de responsabilidad, la capacidad de adaptarse a los cambios y tolerar la incertidumbre frente a lo ambiguo o desconocido, la flexibilidad para incorporar diferentes perspectivas, la persistencia incluso ante eventuales fracasos y la valoración y soporte de las ideas nuevas, incluyendo la gestión de los recursos necesarios.

Estas condiciones dependen fuertemente de los directivos y, en particular, de su estilo de liderazgo que puede actuar favoreciendo condiciones para la innovación o, contrariamente, inhibiéndola y limitándola (Carballo, 2007). Nutrida evidencia internacional muestra que el liderazgo democrático, participativo, colaborador o transformacional favorece la innovación, el desempeño creativo y, en general, condiciones de cultura y clima organizacional más favorables a ellos (p. ej., Farr y Ford, 1990; Kanter 1983; Mumford et al., 2002; Redmon, Mumford y Teach, 1993; Scott y Bruce, 1994), en particular cuando se lo compara con el liderazgo transaccional (Manz et al., 1989) y, específicamente, en instituciones educativas (Pirela de Faría, 2010).

Los constructos de liderazgo transformacional y transaccional son de larga data y han influenciado notablemente la forma en que se entiende el liderazgo. Fueron propuestos por Burns (1978) como extremos de un polo para distinguir entre liderazgos políticos. Los líderes transformacionales se enfocan en propósitos de largo plazo, con una mirada que resuena en necesidades internas de las personas que los siguen, mientras que los transaccionales se enfocan en las metas de corto plazo y en el intercambio de recursos; los primeros actúan por identificación y convicción, mientras que los segundos, por intercambio o transacción. Bass (1985) propuso que ambos estilos de liderazgo no constituyen extremos de un mismo polo, sino dimensiones independientes, que son conjugadas flexiblemente por los líderes exitosos, y exploró las manifestaciones conductuales de estas formas de liderazgo en las organizaciones: la dimensión transformacional se caracteriza por el carisma — enfatiza la confianza, da seguridad, destaca el compromiso, considera aspectos éticos, se contacta emocionalmente con sus seguidores - ; la motivación inspiradora: ofrece una visión de futuro capaz de entusiasmar, anima a establecer criterios elevados, aporta entusiasmo y ofrece sentido o significado trascendente a las acciones; la estimulación intelectual: desafía las convenciones y supuestos previos, solicita ideas a sus seguidores y les anima a realizar propuestas creativas, toma riesgos; y la consideración personal o individualizada, que trata a sus seguidores como personas individuales, considerando sus propias necesidades y aspiraciones, les escucha, los aconseja y enseña.

Mayor información en http://www.nist.gov/baldrige/about/baldrige_faqs.cfm 
La dimensión transaccional se caracteriza por la recompensa contingente: establece compromisos específicos, ofrece y entrega recompensas por los logros; y por la administración por excepción o correctiva: emprende acciones cuando los resultados no son los esperados. No obstante, esta última característica puede adoptar dos formas cuyos efectos son diferentes: la administración activa, en la cual el líder monitorea los avances y sugiere acciones correctivas antes de que se produzcan los fallos o los resultados sean inadecuados, y la administración pasiva, en la que el líder no interviene hasta que los malos resultados ya se han producido (Hater y Bass, 1988). Bass (1985) también incluye la dimensión ausencia de liderazgo, que refleja el comportamiento de los líderes que no asumen el liderazgo: se resisten a tomar decisiones, vacilan en las acciones a seguir, están ausentes cuando se les requiere. Por la semejanza en sus características y efectos, Bass y Avolio (1994) han considerado la administración por excepción pasiva junto a la ausencia de liderazgo a la hora de medir estas dimensiones, resultando en un modelo tridimensional (liderazgo transformacional, liderazgo transaccional y pasivo-evitador).

Los autores proponen que los líderes exitosos implican tanto elementos transformacionales como transaccionales (Avolio, Bruce y Bass, 1999), lo que ha sido apoyado por la investigación empírica, incluso en Chile. Por ejemplo, Cuadra y Veloso (2007) encontraron que características asociadas a ambos liderazgos se asocian positivamente con características positivas del clima y satisfacción laboral, y que el liderazgo pasivo-evitador no correlaciona o lo hace negativamente con ellos.

Resulta clara la coincidencia entre las características atribuidas al liderazgo transformacional y las condiciones favorables a la innovación, por lo que los resultados empíricos descritos más arriba no sorprenden. No obstante, ellos no han sido replicados en Chile. De hecho, al revisar la literatura disponible solo hemos encontrado un estudio que involucra liderazgo e innovación en contexto de organizaciones empresariales: se trata del estudio de Rodríguez (2010), quien encontró que el liderazgo transformacional impacta positiva y fuertemente sobre la cultura de la innovación; y, en el contexto escolar, un estudio de gran alcance sobre el liderazgo directivo en escuelas chilenas (Carbone et al., 2008) en el que se encontró que los directivos de las escuelas con buenos resultados pedagógicos podían ordenarse en un polo que va de lo participativo a lo autoritario, en tanto la capacidad de innovación era una característica destacable en los que se ubican en este último polo. Siendo un resultado interesante que merece ser explorado, la dimensión liderazgo participativoliderazgo autoritario corresponde a una conceptualización distinta a aquella de la que surgen los liderazgos transformador y transaccional, y por lo tanto este hallazgo no permite valorar la influencia del liderazgo transformador o transaccional en las condiciones para la innovación en las escuelas. Así, pese a la reciente y creciente consideración del liderazgo y evidencia acerca de su influencia sobre los resultados académicos en escuelas chilenas (López, 2008, 2010; Pedraja et al., 2009; Pedraja, Rodríguez y Villarroel, 2012), no hay estudios que se enfoquen específicamente en la relación entre estos tipos de liderazgo y condiciones para la innovación en contextos escolares. En ello se enfoca este trabajo.

\section{METODOLOGÍA}

En un diseño cuantitativo, correlacional, se administró dos instrumentos tipo encuesta, de papel y lápiz, a 127 profesores y profesoras que respondieron voluntariamente a la 
invitación a participar de la investigación. Provenían de 14 establecimientos secundarios particulares subvencionados (privados con financiamiento público) de la ciudad de Iquique, Chile, con cuyos directivos se había tomado contacto previamente para solicitar su autorización para invitar a los profesores a participar. El 58\% de los que completaron la encuesta fueron mujeres, el $72 \%$ titulados del ámbito de las pedagogías (en Chile, profesionales no pedagogos pueden ejercer docencia con una habilitación especial, particularmente en escuelas secundarias de carácter técnico-profesional), y el 17\% tenía algún postgrado; provenían de una variedad de disciplinas y especialidades desde lenguaje o matemáticas, hasta artes, educación física o especialidades técnicas.

El primer instrumento fue el Cuestionario multifactorial de liderazgo, adaptación al español del instrumento desarrollado por Bass y Avolio (1995), ampliamente utilizado en investigación sobre liderazgo, incluso en Chile (p. ej., Rodríguez, 2007). Consta de 45 reactivos, con una escala de respuesta de 0 (nunca) a 4 (siempre), que da una medida para cada uno de los tipos de liderazgo-transformacional, transaccional y pasivo-evitador, cuyas confiabilidades en la presente muestra (coeficiente alpha de Cronbach) fueron 0.95, 0.84 y 0.84 , respectivamente.

El segundo instrumento fue construido por los investigadores para evaluar la presencia de condiciones para la innovación en la organización escolar, extrayendo y adaptando al contexto de la organización escolar, ítems de dos guías sobre innovación y creatividad españolas para la empresa (Lan Ekintza-Bilbao, 2004; Ramis, 2005). El resultado fue un instrumento de 11 ítems con una escala de respuesta de 0 (completamente en desacuerdo) a 4 (completamente de acuerdo), que da cuenta de las siguientes condiciones en la organización: capacidad de adaptación a los cambios, flexibilidad de los directivos para tomar lo mejor de diversas perspectivas, disposición a la ampliación de los conocimientos para la generación de nuevas ideas, tolerancia al fracaso y disposición a destinar recursos para la innovación (tiempo, personas, incentivos). La confiabilidad (coeficiente alpha de Cronbach) para la escala resultante fue, en esta muestra, de 0.86 , y las confiabilidades de las subescalas fluctuaron entre .69 y .73 .

\section{RESULTADOS}

En la Tabla 1 se muestran los estadísticos descriptivos. Como se ve, el tipo de liderazgo transformacional tiene la mayor presencia y el liderazgo pasivo-evitador la presencia más baja $(\mathrm{F}=184.395, \mathrm{gl}=2, \mathrm{p}<.000)$. La media de condiciones para la innovación se ubica ligera pero significativamente por sobre la media de la escala $(\mathrm{t}=12.708, \mathrm{gl}=125, \mathrm{p}$ $<.000$ ), y las diferentes condiciones muestran niveles significativamente diferentes de presencia $(\mathrm{F}=102.993, \mathrm{gl}=4, \mathrm{p}<.000)$, son tolerancia al fracaso y disponibilidad de recursos para la innovación las más bajas. 
Estudios Pedagógicos XLII, Nº 2: 193-205, 2016

LIDERAZGO DIRECTIVO Y CONDICIONES PARA LA INNOVACIÓN EN ESCUELAS CHILENAS: EL QUE NADA HACE, NADA TEME

Tabla 1. Estadísticos descriptivos para tipos de liderazgo y condiciones para la innovación

\begin{tabular}{lcccc}
\hline Variable & Mínimo & Máximo & Media & Desviación típica \\
\hline Liderazgo transformacional & 0.58 & 4.00 & 3.06 & 0.770 \\
Liderazgo transaccional & 0.86 & 4.00 & 2.94 & 0.751 \\
Liderazgo pasivo-evitador & 0.00 & 3.63 & 1.37 & 0.836 \\
Condiciones para la innovación & 1.09 & 4.00 & 2.67 & 0.595 \\
Flexibilidad & 1.00 & 4.00 & 2.88 & 0.680 \\
Adaptación a los cambios & 0.67 & 4.00 & 2.85 & 0.710 \\
Ampliación de conocimientos & 1.00 & 4.00 & 2.68 & 0.720 \\
Recursos para innovación & 0.00 & 4.00 & 1.98 & 1.130 \\
Tolerancia al fracaso & 0.00 & 4.00 & 2.21 & 0.880 \\
\hline
\end{tabular}

En la Tabla 2 se muestra la matriz de correlaciones entre las variables. Como se aprecia, tanto el liderazgo transformacional como el liderazgo transaccional correlacionan positiva y moderadamente con condiciones para la innovación y con cada una de sus dimensiones, mientras que el liderazgo pasivo-evitador correlaciona negativa y moderadamente con condiciones para la innovación y con tres de sus dimensiones, y no correlaciona con otras dos. Los tres tipos de liderazgo, en conjunto, explican el 27,5\% de la varianza de condiciones para la innovación $(\mathrm{R}=.541)$, pero la contribución del liderazgo transaccional es marginal, debido a su alta correlación con liderazgo transformacional, que carga el mayor peso explicativo; este último, en conjunto con liderazgo pasivo-evitador, cuyo efecto es negativo, explican $27,3 \%$ de la varianza.

\section{DISCUSIÓN}

Los resultados son ampliamente concordantes con la literatura disponible, tanto en relación a los liderazgos efectivos como a su relación con la innovación. Es clara la presencia de las dimensiones transformacional y transaccional en los liderazgos percibidos en las escuelas, que contrasta con la escasa percepción de liderazgo pasivo-evitador; la relación entre ambas dimensiones, transformacional y transaccional, es muy estrecha, lo que refuerza la idea de que los líderes exitosos emplean ambos tipos de liderazgo simultáneamente (Avolio et al., 1999; Cuadra y Veloso, 2007). Por su parte, como se esperaba, tanto las dimensiones transformacional como transaccional se correlacionan positivamente con las condiciones para la innovación percibidas en las escuelas, mientras que el liderazgo pasivo-evitador se correlaciona inversamente con las mismas. No obstante, y a pesar que la regresión muestra que la incorporación del estilo pasivo-evitador en la ecuación prácticamente no incrementa la proporción de varianza en las condiciones para la innovación explicada por los estilos transformacional y pasivo-evitador en conjunto, 
Estudios Pedagógicos XLII, Nº 2: 193-205, 2016 LIDERAZGO DIRECTIVO Y CONDICIONES PARA LA INNOVACIÓN EN ESCUELAS CHILENAS: EL QUE NADA HACE,

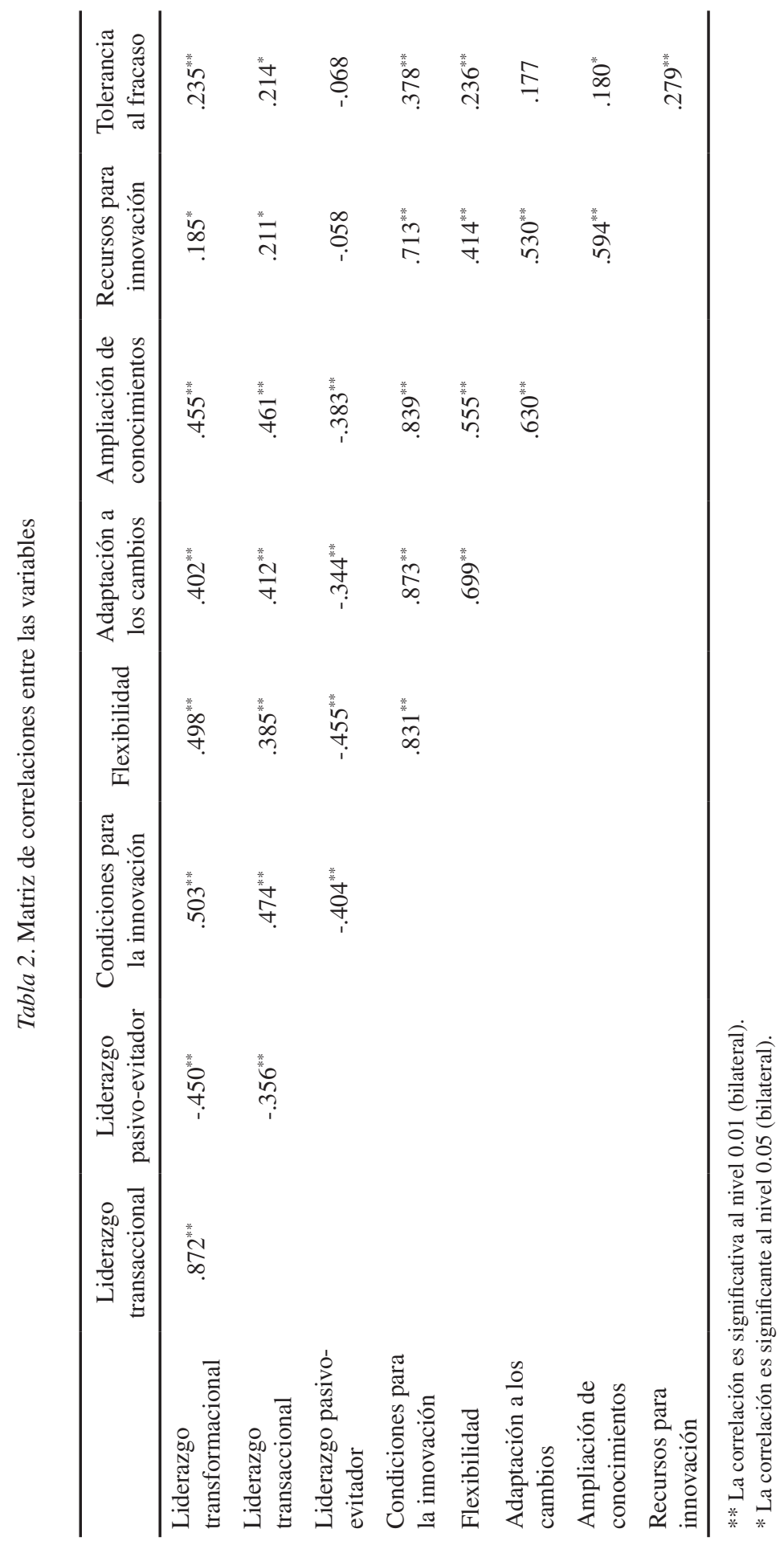


lo que parece confirmar la eventual preeminencia del liderazgo transformacional sobre el transaccional para explicar las condiciones para la innovación como han sugerido varios autores (p. ej., Manz et al., 1989; Rodríguez, 2010), la alta correlación entre ambos tipos de liderazgo hace difícil sostener esa afirmación en este caso, a lo que contribuye la similitud de las correlaciones entre estos dos tipos de liderazgo y las condiciones para la innovación, consideradas por separado.

En síntesis, se confirman varias afirmaciones centrales respecto de liderazgo y condiciones para la innovación: primero, que los liderazgos transformacional y transaccional coexisten en los líderes de las instituciones escolares; segundo, que la presencia de liderazgo activo, tanto transformacional como transaccional, favorece condiciones para la innovación; tercero, que la presencia de liderazgo pasivo-evitador obstaculiza las condiciones para la innovación; cuarto, como corolario, que el liderazgo es un factor relevante a la hora de producir cambios en las escuelas, como han sugerido otros autores (p. ej., Anderson, 2010; Riveros, 2012); y quinto, que la relación entre los liderazgos transformacional y transaccional y las condiciones para innovar necesitan un examen más detallado, probablemente examinando sus dimensiones internas. En este último sentido, varios autores señalan la influencia conjunta de liderazgos transformacionales y participativos u horizontales sobre variables organizacionales, incluyendo innovación (Da Costa et al., 2014; Pons y Ramos, 2012; Tapia et al., 2011); sin embargo, ello no concuerda, en el caso específico de la innovación, con lo encontrado por Carbone et al. (2008) —en cuyo estudio las condiciones para innovación se encontraban en escuelas con estilos autoritarios de liderazgo — ni con nuestros resultados.

La importancia relativa de las diferentes condiciones para la innovación presentes en las escuelas es un dato interesante. Tolerancia y persistencia frente al fracaso y disponibilidad de recursos son las condiciones menos presentes; vale la pena explorar qué podría estar reflejando este hecho. Tolerar y persistir en situaciones de fracaso implica estar dispuesto a equivocarse, a empezar de nuevo, asumir el error y el fracaso como parte de la experiencia de aprendizaje en cualquier contexto; ello no es fácil en una cultura social y escolar marcada por el éxito como condición de subjetividad como es la latinoamericana, en particular la chilena (Montes, 2014; Núñez, 2011; Ospina, Gómez y Rojas, 2014), y por la aversión a la incertidumbre, una de las características que define la cultura nacional (Fernández et al., 1997). La propia cultura escolar, desde su misma identidad pedagógica, lleva décadas (sino centurias) construyéndose sobre la proscripción del error y el signo de la eficacia (De la Torre, 2004). En esas condiciones, ¿es de extrañar que el fracaso sea algo todavía difícil de asumir como parte de la cotidianeidad, condición necesaria para atreverse a innovar?

Disponibilidad de recursos es, con holgura, la más baja de todas las condiciones. Aunque los recursos siempre son escasos, la política educacional chilena ha aumentado sostenida y muy significativamente los recursos económicos disponibles para las escuelas en las últimas décadas (Donoso y Donoso, 2009; Elacqua y Santos, 2013); sin embargo, no basta la existencia de los recursos: además deben estar en un contexto que favorezca su utilización, y en ello podría radicar parte del problema: hay mucha evidencia que muestra que los directivos escolares disponen, en su mayor parte de escasa o nula autonomía, atribuciones o capacidad para gestionar autónomamente los recursos nominalmente disponibles para las escuelas (Ahumada, Sisto y López, 2012; Donoso y Donoso, 2009), y cuando la tienen, pesa sobre ellos la burocratización de la rendición de cuentas, el accountability (Elacqua y Santos, 2013), e incluso a veces la responsabilización por los mismos recursos 
materiales como el equipamiento, que les agregan cargas que no necesariamente están dispuestos a asumir (hay ejemplos anecdóticos respecto a esto que, por razones obvias no están documentados pero que hemos visto directamente, como equipos computacionales o audiovisuales guardados sin uso por temor a tener que responder si se descomponen).

Por otra parte, no se trata sólo de recursos económicos, financieros o materiales; se trata también de otros recursos que pueden ser tan o más escasos o difíciles de administrar y gestionar que los financieros y materiales, como el tiempo, los recursos humanos y las condiciones de trabajo. En este sentido, desde hace varias décadas se viene describiendo en Chile la cultura burocratizadora, controladora y sobrecargada de funciones y tareas del sistema escolar promovida desde la institucionalidad que termina agobiando tanto a profesores como directivos y otros actores escolares, incluso con consecuencias directas sobre el bienestar y la salud de los mismos (Cornejo y Reyes, 2008; López, Assael y Neumann, 1984). Un artículo de revisión destaca que las condiciones organizacionales pueden limitar el uso de prácticas efectivas, desgastando incluso las buenas intenciones de los profesores (Anderson, 2010), y Tapia et al. (2011) señalan que una de las condicionantes más fuertes en la gestión de los directivos escolares en contextos de vulnerabilidad son las condicionantes institucionales para la gestión del tiempo y los recursos. En este contexto, no es difícil caer en un estado de inercia o inacción en que se hace lo justo, lo que se puede o lo mismo de siempre; como un profesor nos dijo en una ocasión para describir la situación, "mejor no hacer nada; el que nada hace, nada teme".

Por su parte, es interesante destacar que flexibilidad y capacidad de adaptación a los cambios son las condiciones para la innovación vistas con mayor presencia por los profesores. Esto parece contradecir la impresión de que los directivos son reacios o resistentes a los cambios, como afirman Gorrochotegui, Vicente y Torres (2014), o que los profesores generan resistencias frente a estrategias de intervención, cambio o mejora hacia la creatividad (Guerrero, 2005); aunque puede ser solo una contradicción aparente, pues lo que declaran los actores suele no coincidir con lo que aprecian eventuales observadores de la situación (Leal et al., 2009). De cualquier manera, la impresión es que se percibe condiciones favorables en aquellas dimensiones más cercanas a los propios actores, y menos en aquellas que, siendo mediadas o vehiculadas por los actores, responden más claramente a condiciones estructurales de la institucionalidad educativa o a elementos enraizados en la cultura escolar o, incluso, en la cultura propia asociada al carácter nacional. Es decir, tomando las palabras de Sisto (2010, p. 33), "La mejora de los establecimientos educativos no depende del director", o, al menos, no solo de él, sino de que las condiciones institucionales y culturales del sistema educativo y la propia escuela lo permitan.

Puesta de este modo la situación, ¿cómo romper esta especie de camisa de fuerza en que se perciben los actores escolares frente al cambio y la innovación, entre las condiciones impuestas por el propio sistema y las eventuales capacidades o posibilidades de sus directivos? Al menos tres pistas pueden adelantarse al respecto. La primera no es una, en realidad, sino dos que pueden ser complementarias: la incorporación de las prácticas reflexivas (Cornejo y Fuentealba, 2008) y las estrategias de aprendizaje colaborativo en sus distintas vertientes (Letor, 2012; Montecinos, Fernández y Madrid, 2011). Ambas son formas de trabajo colectivo que permiten reflexionar y aprender a partir de la práctica cotidiana en el mismo contexto escolar, facilitando el empoderamiento y la movilización de los actores hacia los procesos de mejora autogestionados. Una segunda pista la encontramos en las nuevas formas de entender el liderazgo en la escuela, no como una propiedad del o 
los individuos que tienen responsabilidades directivas, sino como un modo de relacionarse e interactuar de los actores de una comunidad educativa que logra establecer relaciones y desarrollar procesos que resultan productivos y satisfactorios para sus miembros, como han destacado ya algunos autores (Riveros, 2012; Sisto, 2010). Finalmente, la tercera pista atañe al modo en que las innovaciones llegan a o se generan en la escuela y/o son asumidas por esta, lo que puede ser muy moldeado por la forma en que la institucionalidad se relaciona con ellas. Gairín (2000) señala tres modos en que ello puede ocurrir, que corresponden a tres estadios diferentes del desarrollo de la organización. En el primer estadio, la escuela es percibida por sus actores como mera receptora, un contexto en el cual se desarrolla una intervención o innovación, la que existe con cierta independencia de dicho contexto. En el segundo estadio, la organización tiene un planteamiento propio, que puede dialogar con la innovación o intervención; no es solo el contexto, sino que contribuye al proceso. En el tercer estadio, la organización es protagonista, generando su propio proceso, haciéndose cargo de él y sus circunstancias de manera flexible y colectiva, pudiendo evaluar, retroalimentarse y modificar rumbo si es necesario: es la organización que aprende y educa. Sin duda, el trabajo colectivo, reflexivo y colaborativo, el liderazgo distribuido y el desarrollo de la organización hacia la autogestión de su propio aprendizaje, son estrategias que pueden potenciarse y promover mejores condiciones para innovar y mejorar en nuestras escuelas.

\section{REFERENCIAS BIBLIOGRÁFICAS}

Ahumada, L., Sisto, V., \& López, V. (2012). Prácticas de liderazgo de directores y equipos directivos de establecimientos educacionales en Chile. En J. Catalán (Ed.), Investigación orientada al cambio en Psicología Educacional (pp.119-136). La Serena: Ediciones Universidad de La Serena.

Anderson, S. (2010). Liderazgo Directivo: Claves Para Una Mejor Escuela. Psicoperspectivas. Individuo y sociedad, 9(2), 34-52.

Avolio, B., Bruce, J., \& Bass, B. (1999). Re-examinig the components of transformational and transactional leadership using the Multifactor Leadership Questionnaire. Journal of Occupational and Organizational Psychology, 72, 441-462.

Barraza, A. (2005). Una conceptualización comprehensiva de la innovación. Innovación Educativa, 5(28), 19-31.

Barraza, A. (2007). Innovación Educativa. México: Universidad Pedagógica de Durango.

Bass, B. (1985). Leadership and performance beyond expectations. New York: Free Press.

Bass, B., \& Avolio, B. (1994). Improving organizational effectiveness through transformational leadership. Thousand Oaks, CA: Sage.

Bass, B., \& Avolio, B. (1995). The multifactor leadership questionnaire - 5x short form. Redwood: Mind Garden.

Blanco R., \& Messina, G. (2000). Estado del arte sobre las innovaciones educativas en América Latina. Santiago de Chile: Convenio Andrés Bello.

Burns, J. (1978). Leadership. Nueva York: Harper \& Row.

Carballo, R. (2007). Liderazgo e Innovación. Recuperado desde: http://www.robertocarballo. com/2007/01/24/liderazgo-e-innovacion/

Carbone, R., Olguín J., Ostoic, D., Ugalde, P., \& Sepúlveda, L. (2008). Situación del Liderazgo Educativo en Chile. Santiago: Universidad Alberto Hurtado.

Cornejo, R., \& Fuentealba, R. (2008). Prácticas reflexivas para la formación profesional docente: ¿qué las hace eficaces? Santiago de Chile: Ediciones UCSH.

Cornejo, R., \& Reyes, L. (2008). La cuestión docente. Chile: experiencias organizacionales y acción 
colectiva de profesores.. Buenos Aires: Fundación Laboratorio de Políticas Públicas.

Cruz, A. (2008). La Ruta de la innovación en Chile. Revista Observatorio Económico, Universidad Alberto Hurtado, 12, 3-4.

Cuadra, A., \& Veloso, C. (2007). Liderazgo, Clima y Satisfacción Laboral en las Organizaciones. Universum, 22(2), 40-56.

Da Costa, S., Páez, D., Sánchez, F., Gondim S., \& Rodríguez, M. (2014). Factores favorables a la innovación en las organizaciones: una integración de meta-análisis. Journal of Work and Organizational Psychology, 30, 67-74.

De la Torre, S. (2004). Aprender de los errores. El tratamiento didáctico de los errores como estrategias innovadoras. Buenos Aires: Editorial Magisterio del Río de La Plata.

Donoso, S., \& Donoso, G. (2009). Políticas de gestión de la educación pública escolar en Chile (1990 -2010): Una evaluación inicial. Ensaio: Avaliação e Políticas Públicas em Educação, 17(64), 421-448.

Elacqua, G., \& Santos, H. (2013). Preferencias reveladas de los proveedores de educación privada en Chile. El caso de la Ley de Subvención Escolar Preferencial. Gestión y Política Pública, 22(1), $85-129$.

Farr, J. L., \& Ford, C. M., (1990). Individual Innovation. In M.A. West, \& J.L. Farr (Eds.), Innovation and Creativity at Work. Psychological and Organizational Strategies (pp. 63-80). Chichester: Wiley.

Fernández, D., Carlson, D., Stepina, L., \& Nicholson, J. (1997). Hofstede's country classification 25 years later. The Journal of Social Psychology, 137(1), 43-54.

Ferrando, M., \& Granero, J. (2005). Calidad total: Modelo EFQM de excelencia. Madrid: Fundación CONFEMETAL.

Gairín, J. (2000). Cambio de cultura y organizaciones que aprenden. Educar, 27, 31-85.

García, S. (2008). La innovación como reto directivo. Documentos a debate, IDOE-Instituto de Dirección y Organización de Empresas, Universidad de Alcalá, 18, 1-4.

Gorrochotegui, A., Vicente, I., \& Torres, G. (2014). Evaluación de un proceso de coaching en directivos y su impacto en el clima escolar. Revista Educación y Educadores, 17, (1), 111-131.

Guerrero, P. (2005). Estudio de las resistencias de los profesores a una estrategia para el desarrollo de la creatividad en tres unidades educativas. Psykhe, 14(1), 31-45.

Hater, J., \& Bass, B. (1988). Superiors' evaluations and subordinates' perceptions of transformational and transactional leadership. Journal of Applied Psychology, 73, 695-702.

Horn, A., \& Marfán, J. (2010). Relación entre liderazgo educativo y desempeño escolar: revisión de la investigación en Chile. Psicoperespectivas. Individuo y sociedad, 9(2), 82-104.

Kanter, R. (1983). The Change Masters. New York: Simon \& Shuster.

Lan Ekintza-Bilbao, (2004). Guía práctica sobre la innovación y la creatividad. Bilbao: Ayuntamiento de Bilbao.

Leal, F., Gianonni, M., Escares, V., \& Tapia, P. (2009). "Del dicho al hecho”: Entre el discurso y la práctica de los educadores. Trabajo presentado en las IX Jornadas Internacionales de Psicología Educacional, Valparaíso, Chile.

Letor, C. (2012). Desarrollar trabajo colaborativo dentro del centro educativo: un análisis desde una perspectiva organizacional. En J. Catalán, Investigación orientada al cambio en Psicología Educacional (pp. 97-118). La Serena: Universidad de La Serena.

López, G., Assael, J., \& Neumann, E. (1984). La cultura escolar: ¿Responsable del fracaso?. Santiago de Chile: P.I.I.E.

López, P. (2008). Modelo de liderazgo para una dirección efectiva. En S. Garay, P. López y O. Maureira (Eds.), Perspectivas de gestión para la innovación y el cambio educativo (pp. 107134). Santiago: Ediciones Universidad Católica Silva Henríquez.

López, P. (2010). El componente liderazgo en la validación de un modelo de gestión escolar hacia la calidad. Educação e Pesquisa, 36(3), 779-794. 
Manz, C., Barstein, D., Hostager, T., \& Shapiro, G., (1989). Leadership and Innovation: A Longitudinal Process View. In A. Van de Ven, H.L. Angle, \& M.S. Poole (Eds.), Research on the Management of Innovation. The Minnesota Studies (pp. 613-636). New York: Harper and Row.

Montecinos, C., Fernández, M., \& Madrid, R. (2011). Desarrollo de conocimiento experto adaptativo en los docentes: Una aproximación desde el aprendizaje colaborativo entre pares. En J. Catalán (Ed.), Psicología Educacional: Proponiendo Rumbos, Problemáticas y Aportaciones (pp. 217244). La Serena, Chile: Editorial Universidad de La Serena.

Montes, F. (2014, mayo). Esta sociedad está enferma de exitismo. Revista Caras, edición de 8 de mayo de 2014. Recuperado desde http://www.caras.cl/sociedad/fernando-montes-esta-sociedadesta-enferma-de-exitismo/

Mumford, M., Scott, G., Gaddes, B., \& Strange, J. (2002): Leading Creative People: Orchestrating Expertise and Relationships. The Leadreship Quaterly, 13, 705-750.

Núñez, C. (2011). La producción social del sujeto emprendedor como política de subjetivación en la formación laboral en Chile. Ponencia al XXXIII congreso SIP, Colombia, Medellín.

Núñez, I., Weinstein, J., \& Muñoz, M. (2010). ¿Posición Olvidada? Una mirada desde la normativa a la historia de la dirección escolar en Chile. Psicoperspectivas. Individuo y sociedad, 9(2), 53-81.

Ospina, C., Gómez, M., \& Rojas, W. (2014). La constitución de la subjetividad en la educación contable: del proceso implícito a la visibilización de sus impactos. Cuadernos de Contabilidad, 15(37), 187-211.

Pedraja, L., Rodríguez, E., Barreda, M., Sagredo, O., \& Segovia, C. (2009). Estilos de liderazgo y resultados del sistema de medición de la calidad de la educación: un estudio empírico en los colegios básicos de la ciudad de Arica-Chile. Revista chilena de ingeniería, 17(1), 21-26.

Pedraja, L., Rodríguez, E., \& Villarroel, C. (2012). Los estilos de liderazgo y la gestión de recursos humanos: una reflexión acerca del impacto sobre el desempeño de los estudiantes en la educación media. Revista chilena de ingeniería, 20(3), 376-385.

Pirela de Faría, L. (2010). Liderazgo y cultura organizacional en instituciones de Educación Básica. Revista Venezolana de Gerencia, 15(51), 486-503.

Pons, F., \& Ramos, J. (2012). Influencia de los Estilos de Liderazgo y las Prácticas de Gestión de RRHH sobre el Clima Organizacional de Innovación. Revista de Psicología del Trabajo y de las Organizaciones, 24(2), 81-98.

Ramis, J. (2005). Guía práctica de la innovación para PYMES. Valencia: Anetcom.

Redmond, M., Mumford, M., \& Teach, R. (1993). Putting Creativity to Work: Effects of Leader Behavior on Subordinate Creativity. Organizational Behavior and human Decision Processes, 5, 120-151.

Restrepo, J., \& Restrepo, M. (2012) Cinco desafíos en el ejercicio del liderazgo en los rectores de colegios. Educación y Educadores, 15(1), 117-129.

Riveros, A. (2012) La distribución del liderazgo como estrategia de mejoramiento institucional. Educación y Educadores, 15(2), 289-301.

Rodríguez, E. (2007). Gestión del conocimiento y eficacia de las organizaciones. Un estudio empírico en instituciones públicas. Interciencia: Revista de ciencia y tecnología de América, 32(12), 820-816.

Rodríguez, E. (2010). Estilos de liderazgo, cultura organizativa y eficacia: un estudio empírico en pequeñas y medianas empresas. Revista de Ciencias Sociales,16(4), 629-641.

Schwab, K. (2013). The Global Competitiveness Report, 2013-2014: Full Data Edition. Génova: World Economic Forum.

Scott, S., \& Bruce, R. (1994). Determinants of Innovative Behavior A Path Model of Individual Innovation in the Workplace. Academy of Management, 37, 580-607.

Sisto, V. (2010). La mejora de los Establecimientos Educativos no depende del Director. Reflexiones críticas de los "otros expertos" en torno a los cambios en la política educativa chilena. Docencia, $42,33-45$. 
Estudios Pedagógicos XLII, N²: 193-205, 2016 LIDERAZGO DIRECTIVO Y CONDICIONES PARA LA INNOVACIÓN EN ESCUELAS CHILENAS: EL QUE NADA HACE, NADA TEME

Tapia, C., Becerra, S., Mansilla, J., y Saavedra, J. (2011). Liderazgo de los directivos docentes en contextos vulnerables. Educación y Educadores, 14(2), 389-409.

Turbay, M. J. (2013). Liderazgo e innovación organizacional. Psicología desde el Caribe, 30(1), vii-ix. 
